\title{
Symmetry-breaking instabilities of generalized elliptical solitons
}

\author{
Yuan Yao Lin* and Ray-Kuang Lee \\ Institute of Photonics Technologies, National Tsing-Hua University, 101, Section 2, Kuang-Fu Road, \\ Hsinchu City 300, Taiwan \\ *Corresponding author: d928103@oz.nthu.edu.tw
}

Received March 10, 2008; revised April 25, 2008; accepted April 26, 2008; posted May 21, 2008 (Doc. ID 93658); published June 13, 2008

Elliptical solitons in 2D nonlinear Schödinger equations are studied numerically with a more-generalized formulation. New families of solitons, vortices, and soliton rings with elliptical symmetry are found and investigated. With a suitable symmetry-breaking parameter, we show that perturbed elliptical solitons tend to move transversely owing to the existences of dipole excitation modes, which are totally suppressed in circularly symmetric solitons. Furthermore, by numerical evolutions we demonstrate that elliptical vortices and soliton rings collapse into a pair of stripes and clusters, respectively, revealing the experimental observations in the literature. (C) 2008 Optical Society of America

OCIS codes: $190.3270,190.4420,190.6135$.

Symmetry-breaking instabilities residing in a nonlinear system govern the formation of solitary waves in various areas of physics and manifest the nature of most nonlinear wave systems. One example is the transverse instabilities of spatial optical solitons in nonlinear Kerr media [1], associated with the growth of transverse modulations of quasi-one-dimensional bright and dark soliton stripes for both focusing [2] and defocusing [3] nonlinearities. In general, a 2D optical soliton system is modulationally unstable [4]. For optical solitons in silica fibers, the modulation instabilities were studied for different radial dimensions of the fiber core [5]. Then, circularly symmetric vortices are investigated by their azimuthal instabilities for both Kerr [6] and saturable nonlinearities $[7,8]$. In particular, this kind of symmetry-breaking instability turns a circular soliton to collapse and vortices to shrink or become soliton clusters, depending on the vortice power and the saturation power [8].

Elliptical symmetry, in contrast to the degenerated circular one, is more generalized and flexible in the real world, where perfect circular symmetry is rarely achieved. Optical waves within elliptical shapes are obtained in a laser cavity with a special designed cavity [9] that supports exact cavity modes as a InceGaussian mode rather than a typical HermiteGaussian mode. Recently, elliptical solitons are proposed in strong nonlocal media [10,11], a fact that supports similar solutions, such as linear InceGaussian beams [9]. Experimental observations of elliptical solitons are also reported not only in nonconventionally biased photorefractive crystals [12] but also in nonlinear media with thermal-induced nonlocality [13]. Here we further study solitons in Kerr nonlinear media with a more-generalized formulation and analyze the corresponding modulation instabilities for families of elliptical solitons, soliton rings, and vortices. In additional to radial symmetric modes, we show that the dipole excitation mode becomes a dominant component in the elliptical modulation instability spectrum and tends to move elliptical solitons transversely, instead of circularly.
Evolution of elliptical shaped soliton-rings and vortices reveals interesting nonlinear dynamics and shows the evidence that elliptical solitary waves play an significant transition between Laguerre and Hermite soliton clusters [14].

We consider the propagation of an optical beam in a Kerr nonlinear medium described by the normalized 2D nonlinear Schrödinger equation (NLS) in elliptical coordinates $(\mu, \theta, z)$ [9], $i \partial \Psi / \partial z+1 / 2 \Delta_{\perp} \Psi$ $+|\Psi|^{2} \Psi=0$, where $\mu \geq 0$ and $\theta \subset[0,2 \pi]$ are radial and angular elliptical variables, i.e., $\Delta_{\perp}$ $=1 / l^{2}\left(\cosh ^{2}(\mu)-\cos ^{2}(\theta)\right)\left[\partial^{2} / \partial \mu^{2}+\partial^{2} / \partial \theta^{2}\right]$, with the slowly varying electric-field envelope function, $\Psi$ $=\Psi(\mu, \theta ; z)$, and the semifocal separation of elliptical cylinder coordinate, $l$. If $l \rightarrow 0$, the elliptical coordinate is reduced to a cylindrical symmetric one; while $l \rightarrow \infty$, the elliptical coordinate turns into a Cartesian one. We employ separation of variables and a variational approach $[6,15]$ to obtain the soliton solution with the ansatz $\Psi=U(\mu) V(\theta) \exp (i \beta z)$, where $U(\mu)$ is a localized radial function subjected to $U(\infty)=0$ and $V(\theta)=V(2 \pi+\theta)$ is an angular function. A set of nonlinear equations can be derived by minimizing the averaged Lagrangian of NLS as

$$
\begin{aligned}
& -\left[\beta f_{1}(\theta)+\frac{f_{2}(\theta)}{2}\right] V+\frac{\partial}{\partial \theta}\left\{\frac{f_{3}(\theta)}{2} \frac{\partial V}{\partial \theta}\right\}+f_{4}(\theta)|V|^{2} V=0, \\
& -\left[\beta g_{1}(\mu)+\frac{g_{2}(\mu)}{2}\right] U+\frac{\partial}{\partial \mu}\left\{\frac{g_{3}(\mu)}{2} \frac{\partial U}{\partial \mu}\right\}+g_{4}(\mu)|U|^{2} U \\
& \quad=0,
\end{aligned}
$$

where $g_{i}(\mu), f_{i}(\theta), i=1,2,3,4$ are functions of integral parameters depending on the selection of solution pair, $U(\mu)$ and $V(\theta)$. By solving Eqs. (1) and (2), a selfconsistent solution of elliptical solitons can be obtained. Figures 1(a) and 1(b) show the radial function $U$ and the angular function $V$ for a fundamental soliton in an elliptical coordinate with $l=0.05$ (solid 

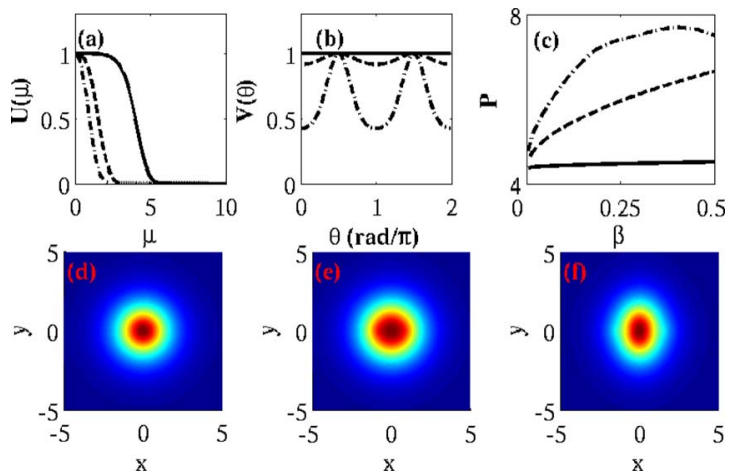

Fig. 1. (Color online) (a) Radial function $U(\mu)$, (b) angular function $V(\theta)$, and (c) bifurcation curves of elliptical solitons at $\beta=0.5$ for different semifocal separations: $l=0.05$ (solid curve), $l=0.7$ (dashed curve), and $l=1.4$ (dotted curve). The corresponding wave functions $\Psi$ are given separately in (d) for $l=(d) 0.05$, (e) 0.7, and (f) 1.4 .

curve), $l=0.7$ (dashed curve), and $l=1.4$ (dotted curve) at $\beta=0.5$. The corresponding soliton solutions $\Psi$ are plotted in Figs. 1(d)-1(f). Clearly, it is seen that a larger semifocal separation $l$ makes the radial function more localized and strengthens the modulation depth of the angular function, with the peak value situated in $\theta=\pi / 2$ and $3 \pi / 2$. Additionally, the bifurcation curves illustrated in Fig. 1(c) also demonstrate that elliptical solitons increase their power $P$ as the semifocal separation increases. It is also seen that when $l$ approaches zero, solitons degenerate to circular-symmetric ground state, which is located at the stability border of the 2D NLS [4]; the elliptical ones are excited states that are expected to be unstable.

The modulation instability of elliptical solitons is investigated through standard linear-stability analysis, with the introduction of perturbed solution in the form of $\Psi=e^{i \beta z}[U(\mu)+\varepsilon \Delta U(\mu ; z)][V(\theta)+\varepsilon \Delta V(\mu ; z)]$, where $U$ and $V$ are the stationary solutions of Eqs. (1) and (2), $\varepsilon \ll 1$ is a small perturbation parameter, and $\Delta U(\mu ; z)$ and $\Delta V(\theta ; z)$ are perturbed envelope functions.

In Fig. 2, we define the elliptical modulation instabilities as the field growth rate with respect to the semifocal separations $l$, which is related to the eccentricity of the soliton solution by the preconditions [16]. For a fixed wave vector, $\beta=0.5$, we show that the modulation instabilities of a $2 \mathrm{D}$ elliptical soliton are excited by three unstable eigenmodes. These excitation modes are identified as a radial symmetric and two dipole modes, corresponding to solid (A), dashed (B), and dashed-dotted (C) curves, respectively. From the modulation instability spectrum, we find that one can suppress the instability of radial symmetric eigenmodes when preconditioned with a cutoff semifocal separation at $l=1.7$ and can distinguish the region where radial modes or dipole modes become dominant. Moreover, elliptical solitons perturbed by unstable dipole noise are driven to the direction in which the dipoles are aligned as it evolves and eventually collapse at the location apart from the origin, as shown in Figs. 2(b)-2(e). At $l=1.2$, simultaneous perturbation of two unstable modes causes the soli-
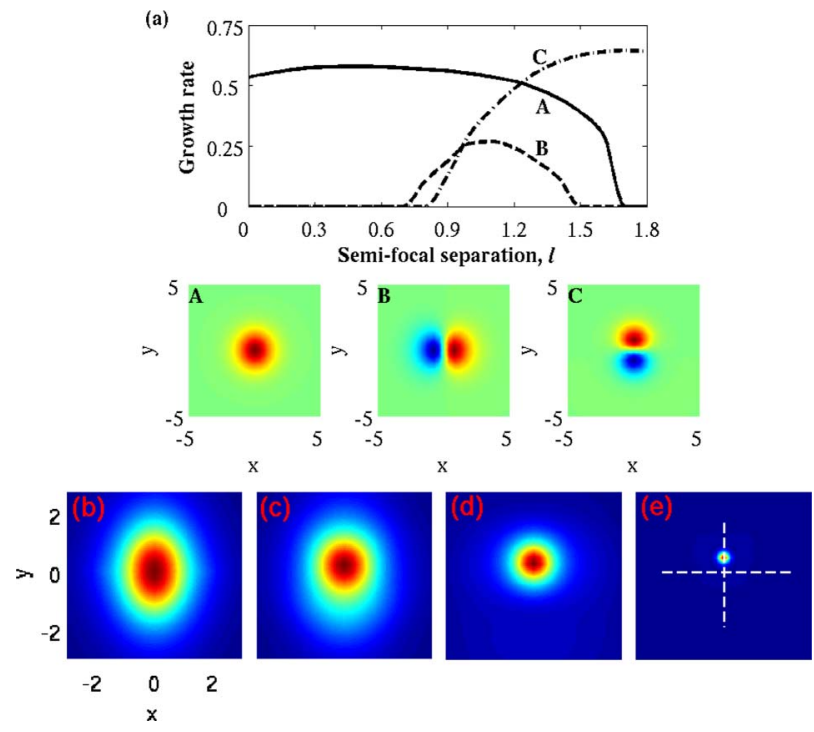

Fig. 2. (Color online) (a) Modulation instabilities of elliptical solitons for different semifocal separations, $l$. A, B, and $\mathrm{C}$ indicate three different eigenfunctions that are shown in the second row with $l=1$.3. Snapshots of propagation of the fundamental elliptical soliton simulated by the NLS are shown at $z=$ (b) 0 , (c) 0.5 , (d) 2 , and (e) 10 , with the semifocal separation $l=1.7$.

ton to collapse and shortens the transverse displacement.

On the basis of the generalized method developed in this work, another two families found in these elliptically preconditioned equations [Eqs.(1) and (2)] are a vortex solution that completes a $2 \pi$ phase change in the azimuthal direction and a ringlike soliton solution with an uniform azimuthal phase. In Figs. 3(a) and 3(b) we show the radial and angular functions of elliptical vortices for different semifocal separations. Similar to the fundamental elliptical solitons, the radial functions are more localized, and the corresponding angular function experiences a stronger modulation depth as $l$ increases. Yet the ra-

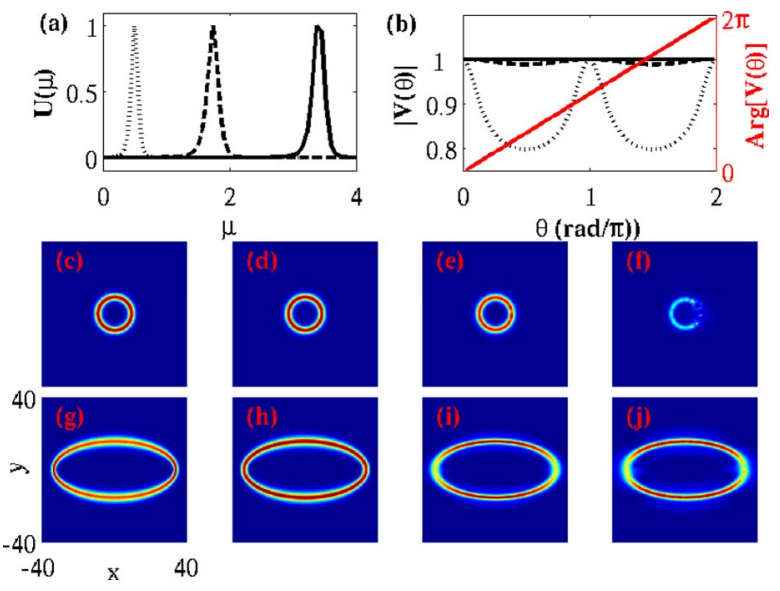

Fig. 3. (Color online) (a) Radial function $U(\mu)$ and (b) angular function $V(\theta)$ (black for envelopes; the diagonal line is for phases) of an elliptical vortice at $\beta=0.5$ for different semifocal separations: $l=1$ (solid curve), $l=5$ (dashed curve), and $l=30$ (dotted curve); evolution of elliptical vortices for (c)-(f) $l=1$ and (g)-(i) $l=30$ at the corresponding distances $z=0,1,4$, and 6.7 . 


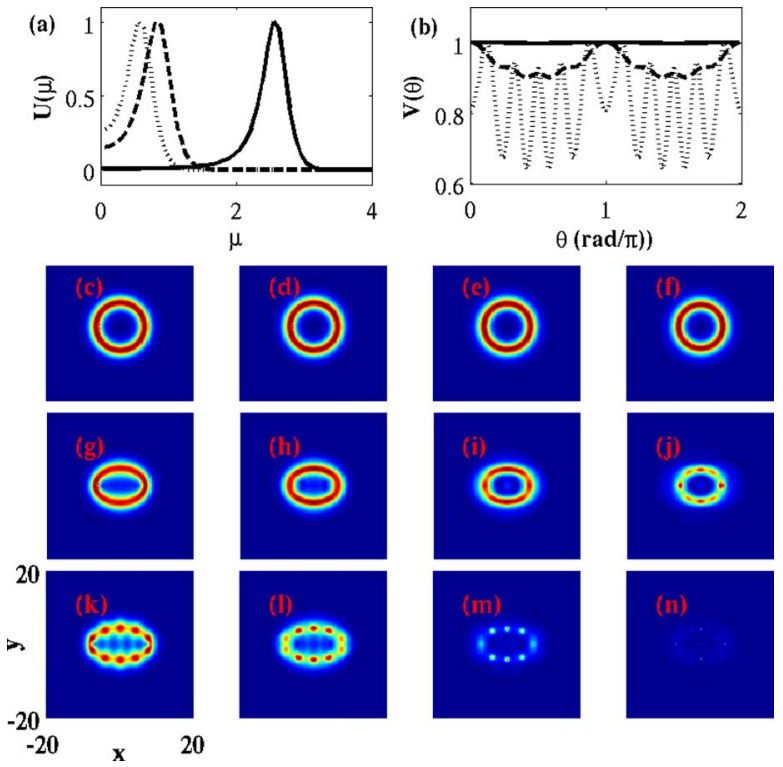

Fig. 4. (Color online) Radial function $U(\mu)$ (a) and angular function $V(\theta)$ (b) of elliptical soliton rings at $\beta=0.5$ are shown in solid, dashed, and dotted curves for $l=1,5$, and 7 , respectively. Evolution of elliptical soliton rings given $l=1$ $[(\mathrm{c})-(\mathrm{f})], l=5[(\mathrm{~g})-(\mathrm{i})]$, and $l=7[(\mathrm{j})-(\mathrm{n})]$ in a window of size $40 \times 40$ are plotted at $z=0,1.5,3$, and 4.5 .

dial functions are pushed to $\mu \sim 0$, and the corresponding angular function has its pinnacles at $\theta=0$ and $\pi$.

To see the nonlinear dynamics and modulation instability of elliptical vortices, we study numerically the evolution of elliptical vortices described by NLS. Figures 3(c)-3(i) compare the modulation instability of elliptical vortices with a fixed propagation constant $\beta=0.5$ but different semifocal separations $(l=1$ and $l=30$ ). We observe that at $z=4$, Fig. 3(i), the strong elliptical instability tears the vortex with $l=30$ into two curved stripes, similar to the "two half-moons" observed experimentally by Anastassiou et al. [8], who attributed the phenomenon to the anisotropy of photorefractive crystal. Our work further reveals that the phenomenon is strongly related to the geometry of prepared vortices, even in perfect material isotropy. In reality, it should be easier to generate elliptical vortices, owing to imperfection induced naturally by symmetry breakings. Then, when this elliptical vortex comes to $z=6.7$, Fig. $3(\mathrm{j})$, clusters riding on each stripe are formed as a result of transverse instability [2]. In contrast, an elliptical vortex with $l$ $=1$ stays stable over $z=4$, Fig. 3(e), and evolves into clusters at $z=6.7$, Fig. 3(f) $[7,8]$. Although both elliptical vortices with $l=1$ and $l=30$ end up with soliton clusters locating at nodal points of the Ince-Gaussian mode $[9,10]$, their geometric arrangements are between two limits of soliton clusters; i.e., with $l=1$ the elliptical vortex transforms into Laguerre soliton clusters, and with $l=30$ it becomes Hermite soliton clusters. Consequently, these facts support the theory that the elliptical solitary wave is a more-general family in nonlinear media.

Elliptical soliton rings are illustrated by their radial and angular functions in Figs. 4(a) and 4(b); the angular functions are modulated by two frequencies in $\theta$ and behave like soliton clusters riding on the elliptical orbit. By comparing the evolution of elliptical soliton rings with $l=1$ [Figs. 4(c) $-4(\mathrm{f})$ ], $l=5$ [Figs. $4(\mathrm{~g})-4(\mathrm{j})$ ], and $l=7$ [Figs. $4(\mathrm{k})-4(\mathrm{n})$ ], we discover that the larger semifocal separation deteriorates the elliptical soliton ring then brings it fast to soliton clusters with an Ince-mode topology, and finally into collapse.

In short, we find families of elliptical soliton, soliton ring, and vortice solutions with a more-general formulation and investigate their modulation instabilities. For elliptical solitons, we demonstrate that by introducing an elliptical coordinate, the instability of radial symmetric eigenmodes is suppressed, and higher excitations, such as dipole modes, tend to move elliptical solitons transversely and become dominant. For elliptical-shaped vortices and soliton rings, we show by evolutions that the semifocal separation presides in the formation of soliton clusters as a consequence of transverse instability, which eventually leads to collapse of vortices and soliton rings.

The research is supported by the National Science Council of Taiwan under contracts 95-2112-M-007058-MY3 and NSC-95-2120-M-001-006.

\section{References}

1. Y. S. Kivshar and D. E. Pelinovsky, Phys. Rep. 331, 117 (2000); and references therein.

2. V. E. Zakharov and A. M. Rubenchik, Sov. Phys. JETP 38, 494 (1974).

3. E. A. Kuznetsov and S. K. Turitsyn, Sov. Phys. JETP 67, 1583 (1988).

4. J. J. Rasmussen and K. Rypdal, Phys. Scr. 33, 481 (1986).

5. N. N. Akhmediev, V. I. Korneev, and R. F. Nabiev, Phys. Rev. A 46, 430 (1992).

6. A. S. Desyatnikov, A. A. Sukhorukov, and Y. S. Kivshar, Phys. Rev. Lett. 95, 203904 (2005).

7. D. V. Skryabin and W. J. Firth, Phys. Rev. E 58, 3916 (1998).

8. C. Anastassiou, C. Pigier, M. Segev, D. Kip, E. D. Eugenieva, and D. N. Christodoulides, Opt. Lett. 26, 911 (2001).

9. M. A. Bandres and J. C. Gutiérrez-Vega, J. Opt. Soc. Am. A 21, 873 (2004).

10. S. Lopez-Aguayo and J. C. Gutierrez-Vega, Opt. Express 15, 18,326 (2007).

11. D. Deng and Q. Guo, Opt. Lett. 32, 3206 (2007).

12. P. Zhang, J. Zhao, C. Lou, X. Tan, Y. Gao, Q. Liu, D. Yang, J. Xu, and Z. Chen, Opt. Express 15, 536 (2007).

13. O. Katz, T. Carmon, T. Schwartz, M. Segev, and D. N. Christodoulides, Opt. Lett. 29, 1248 (2004).

14. D. Buccoliero, A. S. Desyatnikov, W. Krolikowski, and Y. S. Kivshar, Phys. Rev. Lett. 98, 053901 (2007).

15. B. A. Malomed, in Progress in Optics, E. Wolf, ed. (North-Holland, 2002), Vol. 43, p. 71.

16. A. Lifschitz, Phys. Fluids 7, 1626 (1995). 